\title{
Laser-produced uranium plasma characterization and Stark broadening measurements
}

Cite as: Phys. Plasmas 26, 093103 (2019); https://doi.org/10.1063/1.5099643

Submitted: 11 April 2019 . Accepted: 13 August 2019. Published Online: 03 September 2019

M. Burger, P. J. Skrodzki (D) I. Jovanovic, M. C. Phillips, and S. S. Harilal (D)

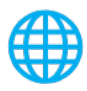

View Online

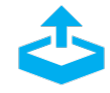

Export Citation

\section{ARTICLES YOU MAY BE INTERESTED IN}

Plume dynamics and gas-phase molecular formation in transient laser-produced uranium plasmas

Physics of Plasmas 26, 083508 (2019); https://doi.org/10.1063/1.5087704

Proton acceleration due to laser plasma interactions from mass-limited spherical targets Physics of Plasmas 26, 093106 (2019); https://doi.org/10.1063/1.5097630

Effects of hole-boring and relativistic transparency on particle acceleration in overdense plasma irradiated by short multi-PW laser pulses

Physics of Plasmas 26, 093108 (2019); https://doi.org/10.1063/1.5120068

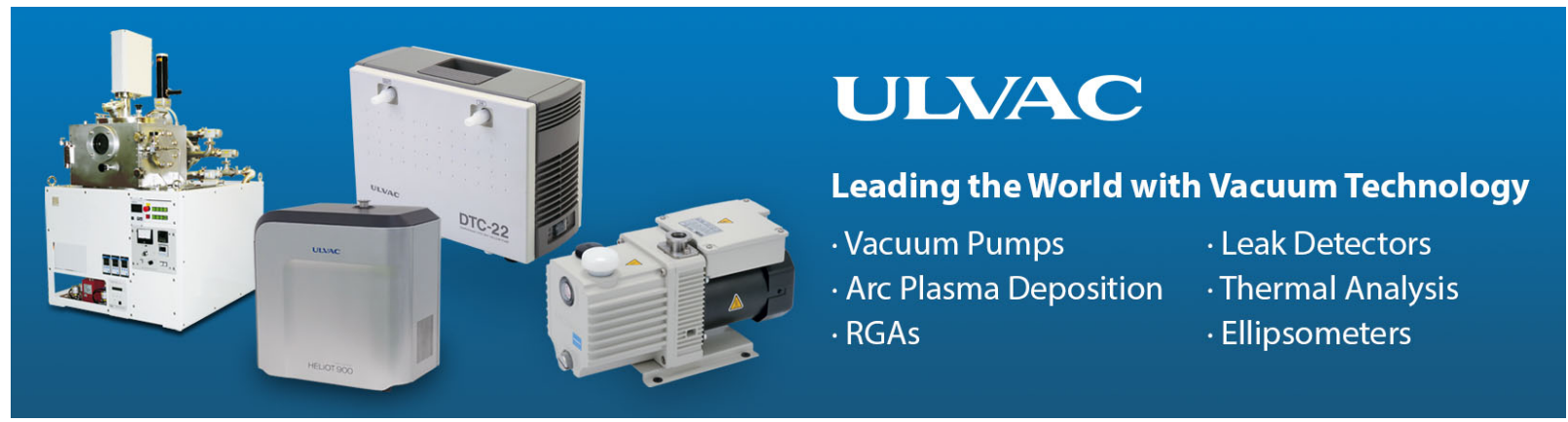




\title{
Laser-produced uranium plasma characterization and Stark broadening measurements
}

\author{
Cite as: Phys. Plasmas 26, 093103 (2019); doi: 10.1063/1.5099643 \\ Submitted: 11 April 2019 • Accepted: 13 August 2019 . \\ Published Online: 3 September 2019
}
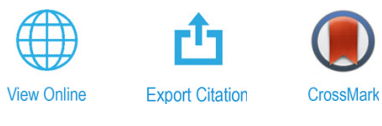

M. Burger, ${ }^{1,2, a)}$ P. J. Skrodzki, ${ }^{1,2}$ (D) I. Jovanovic, ${ }^{1,2}$ M. C. Phillips, ${ }^{3,4,5}$ and S. S. Harilal ${ }^{3, b)}$ (D)

\author{
AFFILIATIONS \\ 'Department of Nuclear Engineering and Radiological Sciences, University of Michigan, Ann Arbor, Michigan 48109, USA \\ ${ }^{2}$ Center for Ultrafast Optical Science, University of Michigan, Ann Arbor, Michigan 48109, USA \\ ${ }^{3}$ Pacific Northwest National Laboratory, Richland, Washington 99352, USA \\ ${ }^{4}$ College of Optical Sciences, University of Arizona, Tucson, Arizona 85721, USA \\ ${ }^{5}$ Opticslah, 2350 Alamo Avenue SE, Suite 280, Albuquerque, New Mexico 87106, USA \\ a)Electronic mail: milosb@umich.edu \\ ${ }^{\text {b) }}$ Electronic mail: hari@pnnl.gov
}

\begin{abstract}
This work reports the spatiotemporal diagnostics of uranium species in plasma plumes produced by nanosecond near-infrared laser pulses in a low-pressure environment. Spatially and temporally resolved emission spectroscopy experiments are combined with the modeling of uranium emission for investigating the dynamics of the plume. The Saha-Eggert equation and Boltzmann plots generated from numerous U I transitions are used to infer temperature. This work also reports the measurements of uranium Stark broadening parameters for U I $499.01 \mathrm{~nm}$ and U II $500.82 \mathrm{~nm}$ transitions. The Stark widths of select U transitions were measured by comparing their linewidths with the broadening of the O I $777.19 \mathrm{~nm}$ line. The electron density was found to be of the order of $10^{16} \mathrm{~cm}^{-3}$, while the temperature was found to be in the range of 3000-9000 K. In addition to enhancing the fundamental understanding of high-Z plasmas in reduced-pressure environments, the knowledge of Stark broadening parameters could improve the modeling capabilities and analytical performance of techniques that rely on emission plasma spectroscopy.
\end{abstract}

Published under license by AIP Publishing. https://doi.org/10.1063/1.5099643

\section{INTRODUCTION}

The ability to rapidly detect and quantify the concentration of actinides in a sample is an important capability for various nuclear applications. For example, in nuclear forensics analysis, it is desired to characterize the material and infer its origin and production history based upon the elemental and isotopic composition. Laser ablation (LA)-based techniques have been demonstrated in this context ${ }^{1-11}$ and shown to exhibit several attractive characteristics, including little to no sample preparation, applicability to a wide range of materials, no requirement of specific sample dimensions, real-time capability, high sensitivity, and feasibility of remote analysis. ${ }^{12}$ LA-optical emission spectroscopy (LA-OES), often referred to as laser-induced breakdown spectroscopy (LIBS), is a powerful technique whereby optical emission from a laserinduced plasma (LIP) is resolved spectrally. ${ }^{12-14}$ Additional analytical benefits can be derived from temporally resolving the LIBS spectra.

Although LIBS is a proven method for elemental detection and analysis, broadening and merging of densely packed spectral lines can make it especially challenging for studying high-Z elements such as uranium and other actinides. In order to understand the processes occurring in laser-induced plasma, it is necessary to evaluate the variation of its thermodynamic parameters. Further research is necessary to foster the capabilities of the LIBS technique toward mature and reliable nuclear material sensing. ${ }^{15-17}$ The interplay of physical and chemical processes in the $\mathrm{U}$ plasma is complex, and limited information is available in the literature pertaining to the spectroscopic coefficients of $U$ transitions such as the ones for Stark widths and shifts. Emission spectroscopy of $U$ plasmas proves challenging because the high- $Z$ analyte yields a congested spectrum consisting of more than $10^{5}$ lines originating from $\sim 1600$ energy levels spanning the UV, visible, and near-IR wavelengths. ${ }^{18-20}$ Moreover, LA of $U$ in reactive environments and the formation of molecules add a large number of additional spectral lines, which often overlap to result in an unresolved broad spectrum. ${ }^{21,22}$ The high density of states in high- $\mathrm{Z}$ molecules produces fine features which interfere with the measurement of the atomic and ionic emission spectra. In such a dense and complex system, the choice of emission lines plays an important role in quantitative analysis. The 
recorded spectral line broadening in LIBS at early times depends on measurement parameters such as the time delay $\left(t_{d}\right)$ after the arrival of the laser pulse and the gate (integration) time.

Important goals for plasma characterization include determining the existence of local thermodynamic equilibrium (LTE) ${ }^{23}$ and the measurements of spatiotemporal evolution of electron temperature $\left(T_{e}\right)$ and electron density $\left(N_{e}\right)$. The mechanism mainly responsible for spectral broadening in LIP is the Stark effect. The Stark broadening becomes dominant ${ }^{24}$ in the spectral line shape formation process in the plasmas where $N_{e} \gtrsim 10^{16} \mathrm{~cm}^{-3}$. The intensity of spectral lines is, on the other hand, strongly influenced by the electron plasma temperature $\left(T_{e}\right)$. It is therefore a high priority to understand the evolution of the magnitude of $N_{e}$ and $T_{e}$ in LIP.

LIBS was previously employed for the detection of $U$. Wachter and Cremers ${ }^{25}$ used LIBS to detect tens of ppm of $U$ as a part of process control in nuclear fuel reprocessing facilities. The role of air ambient pressure on $U$ signal-to-background ratios, line intensities, and FWHMs was investigated previously. ${ }^{26}$ Mechanisms for the $\mathrm{U}$ signal variation under various environmental conditions showed that the persistence of the $U$ ground state population is significantly reduced in the presence of air compared to nitrogen. ${ }^{27}$ LIBS has been successfully utilized for the quantification of $U$ and thorium (Th) in a solution using membrane-based filter paper. ${ }^{28}$ Besides the measurements of elemental abundance, LIBS has also been used for the characterization of U plasma parameters $^{17}$ in the $385.8-391.9 \mathrm{~nm}$ spectral window. However, the uranium LIP literature does not contain the studies of the equilibrium conditions that could lead to theoretical and experimental determination of uranium Stark coefficients. The knowledge of Stark coefficients is required for more comprehensive modeling of physical processes within the plasma and for the improvement of diagnostic and analytic capabilities. ${ }^{29}$ Since the analysis becomes more challenging for high- $Z$ plasma, Stark broadening parameters and shifts of numerous transitions are well documented in the literature for low- $Z$ elements; ${ }^{24,30-32}$ however, very limited information is available for high- $Z$ elements. The numerical modeling of uranium emission spectra is reliant on the accurate knowledge of such broadening coefficients. Spectral simulation may aid toward deeper understanding and deconvolution of underlying oxide spectral features from ionic or atomic emission in the plasma, for example, for $\mathrm{U}$ plasmas in which $\mathrm{U}$ oxide emission is significant and makes the plasma diagnostics more challenging. ${ }^{11,22,33}$

In this work, we assess the validity of equilibrium assumptions in the U LIP under reduced pressure conditions in order to determine the plasma properties, namely, $T_{e}$ and $N_{e}$ and report the Stark broadening parameters of U II $500.82 \mathrm{~nm}$ and U I $499.01 \mathrm{~nm}$ transitions.

\section{EXPERIMENT}

In our LA experimental setup depicted in Fig. 1, a uranium metal target (natural isotopic concentration, $1.0 \times 1.0 \times 0.1 \mathrm{~cm}^{3}$ ) was placed in a chamber which was then evacuated to a pressure of $0.67 \mathrm{~Pa}$ ( 5 mTorr). The chamber was subsequently purged with pure nitrogen (99.999\%) gas. An Nd:YAG laser (Continuum Surelite EX, $1064 \mathrm{~nm}$, $6 \mathrm{~ns}$ ) was focused to a $\sim 1-\mathrm{mm}$ diameter spot with a $15-\mathrm{cm}$ focal length lens; the laser fluence at the target was $\sim 10 \mathrm{~J} \mathrm{~cm}^{-2}$ for the set pulse energy of $75 \mathrm{~mJ}$. The target was translated periodically to prevent target drilling; approximately 20 laser cleaning shots were applied at each new target position in order to remove the uranium oxide layer initially present on the sample surface. LIP emission was imaged through

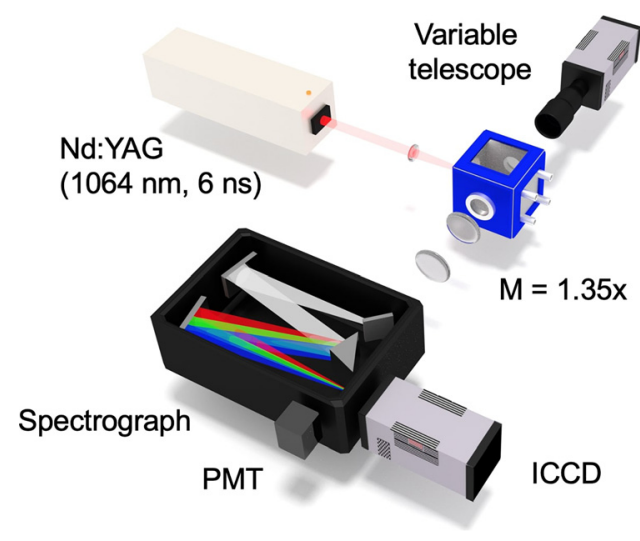

FIG. 1. Setup for ns laser ablation of the uranium target and spectroscopy and imaging of LIP emission.

the quartz window of the chamber with a collection telescope $(1.35 \times$ magnification $)$ onto a $0.5-\mathrm{m}$ Czerny-Turner spectrograph (Acton Spectrapro 2500i) coupled to an intensified charge-coupled device (ICCD, Princeton Instruments PiMAX) and a photomultiplier tube (PMT, Hamamatsu R929, 2-ns rise time). The spectrographICCD detection system provided a maximum spectral resolution of $\sim 35 \mathrm{pm}$ with a 2400 lines/mm grating. The instrumental FWHM with a 1200 lines/mm grating used to record oxygen emission in the nearinfrared spectral region is measured to be $\sim 70 \mathrm{pm}$. The instrument function determination for our detection system was performed using a He-Ne laser and an $\mathrm{Hg}$-Ar lamp by recording the profile of the 632.8-nm laser line and the 404.6-nm mercury line for $1200 \mathrm{l} / \mathrm{mm}$ and $24001 / \mathrm{mm}$ gratings, respectively. We found that the Voigt profile is well suited for modeling our instrumental profile and corresponds to the late time emission spectral profiles of both uranium and oxygen lines. The wavelength and spectral intensity calibration was performed using an Ar-Hg lamp and a calibrated deuterium halogen lamp. Spectroscopic measurements are performed with an entrance slit width of $30 \mu \mathrm{m}$. Since the emission is averaged along the line of sight, the quantitative results should be viewed with caution. The temporal evolution of the selected transitions was recorded using PMT with an entrance slit width of $50 \mu \mathrm{m}$ and an exit aperture width of $100 \mu \mathrm{m}$ and averaged over 16 laser shots. The PMT was connected to a 1-GHz digital storage oscilloscope with $50-\Omega$ termination, for recording the temporal evolution of the selected $U$ transitions. The small translation of the lenses in the imaging telescope allowed for scanning the plasma image on the plane of the spectrograph slit along the direction of plasma expansion. The vertical slit aperture was $\sim 2 \mathrm{~mm}$, and the slit was centered at the laser axis for both multi-channel (spectrally resolved) and single-channel (PMT) measurements.

\section{NUMERICAL MODELING}

To help discriminate the atomic from the ionic and molecular emission, synthetic spectra are generated for U I and U II transitions. The spectral simulations were performed using line parameters from the work by Corliss ${ }^{34}$ and the Palmer Spectral Atlas. ${ }^{19}$ The lines were convolved with a 35-pm FWHM Lorentz function to mimic the instrumental broadening from the detection system. The $1-\mathrm{cm}$ thick slab of plasma with a uniform $U$ density of $10^{15} \mathrm{~cm}^{-3}$ in a temperature 
range of 3000-10000 K was modeled. The simulated spectrum is used to identify U I and U II emissions when the uranium oxide emission may be simultaneously present. ${ }^{35}$

\section{RESULTS}

\section{A. Plasma morphology and expansion}

The morphology of the LIP is an important factor to consider prior to performing the diagnostics of the plasma's thermodynamic parameters. Figure 2 shows the spectrally integrated $(350-800 \mathrm{~nm})$ images obtained using fast gated ICCD photography with the indicated gatewidths. Each image is obtained from a single laser pulse with

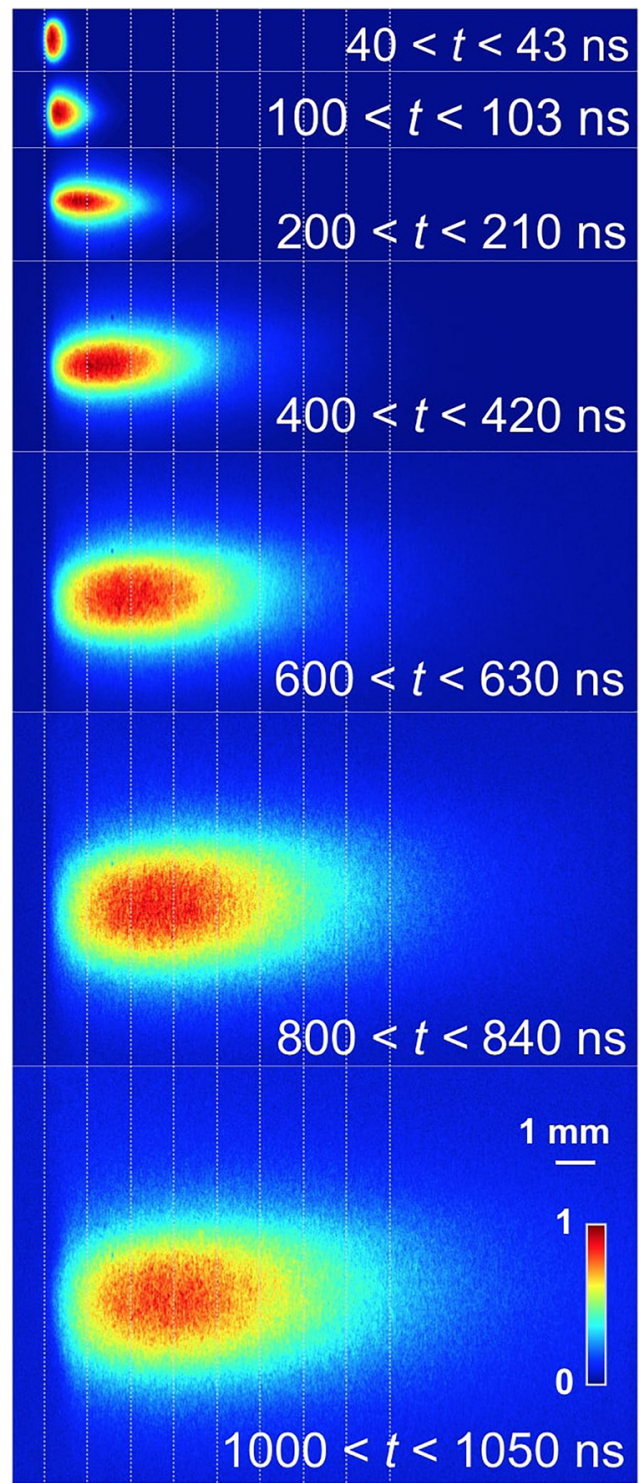

FIG. 2. Time-resolved images of free-expanding LIP. Each image frame is normalized to its maximum intensity. a timing jitter being $<1 \mathrm{~ns}$. The images show the free expansion of the plume into low-pressure $[\sim 0.67 \mathrm{~Pa}$ (5 mTorr)] ambient air. In this pressure range, the rate of plasma expansion is constant and can be predicted by theoretical models and numerical gas dynamics simulations. ${ }^{36}$ The axial (along the laser axis) expansion rate is $\sim 22 \mathrm{~km} / \mathrm{s}$, while the radial (perpendicular to the laser axis) expansion rate is slower, at $\sim 5.0 \mathrm{~km} / \mathrm{s}$ (see Fig. 3 ).

In order to investigate the expansion of a particular species in the plasma, we track in space and time the emission lines of atomic and ionic $\mathrm{U}$ and atomic $\mathrm{O}$. We combine the time-resolved emission profiles for a particular spectral line measured at various distances from the target to form the contours shown in Figs. 4(a)-4(c). The emission profiles were measured using a monochromator-PMT configuration, and the distances were selected by imaging the plasma onto the slit of the monochromator. The contours provide the spatial and temporal particle distribution that can be linked to electron density and excitation temperature. We show emission contours for neutral atomic and ionic $\mathrm{U}$ species and the $\mathrm{O}$ impurity, whose origin is dominantly attributed to the target surface, as evidenced by the expansion dynamics for O shown in Fig. 4(c). The oxygen transitions around $\mathrm{O}$ I $777 \mathrm{~nm}$ originate from a relatively high energy level $(10.74 \mathrm{eV})$ and have transition probabilities of $3.7 \times 10^{7} \mathrm{~s}^{-1}$. From the emission contour plots, we determine the particle velocities by determining the time at which the PMT emission profile peaks at each spatial position. The atomic $U$ species exhibits the slowest axial expansion rate of $\sim 4.0 \mathrm{~km} / \mathrm{s}$, corresponding to a kinetic energy $(K E)$ of $\sim 20 \mathrm{eV}$. The $\mathrm{U}$ ionic species has a greater velocity of $\sim 16 \mathrm{~km} / \mathrm{s}(K E \sim 320 \mathrm{eV})$ and appears to dissipate more quickly via recombination. Assuming that atomic oxygen originates from the target, the species exhibits a velocity of $\sim 13 \mathrm{~km} / \mathrm{s}$ and a similar kinetic energy to atomic $\mathrm{U}$ of $\sim 15 \mathrm{eV}$. Similar kinetic energies of oxygen and uranium species from the target further justify the use of the $\mathrm{O} I$ line for comparison with U-line broadening in the latter determination of Stark widths.

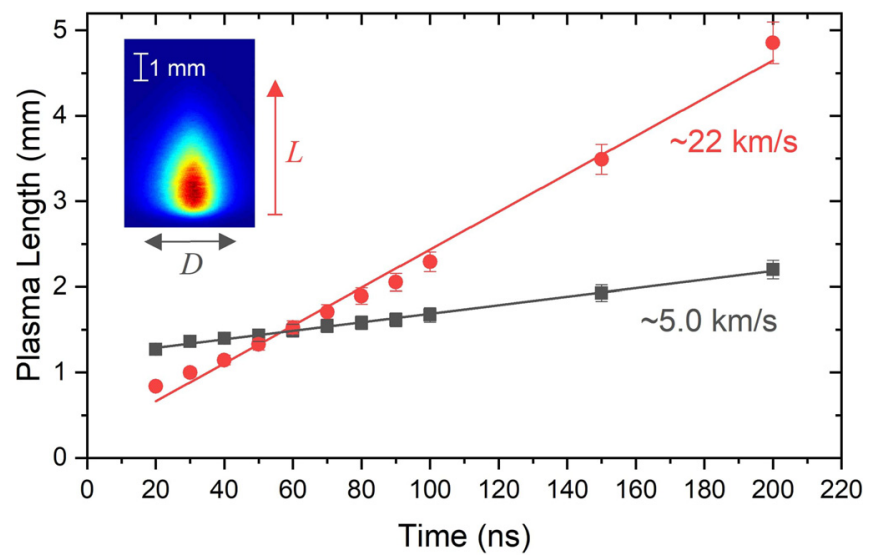

FIG. 3. Estimated expansion velocities of LIP under low-pressure conditions in axial ( $L$, along the laser axis) and radial ( $D$, perpendicular to the laser axis) directions. Distances were determined in the axial direction from the target surface to $5 \%$ of the maximum emission observed in each image. Diameters were determined similarly, using $5 \%$ of the maximum emission in each image as the measurement threshold. 


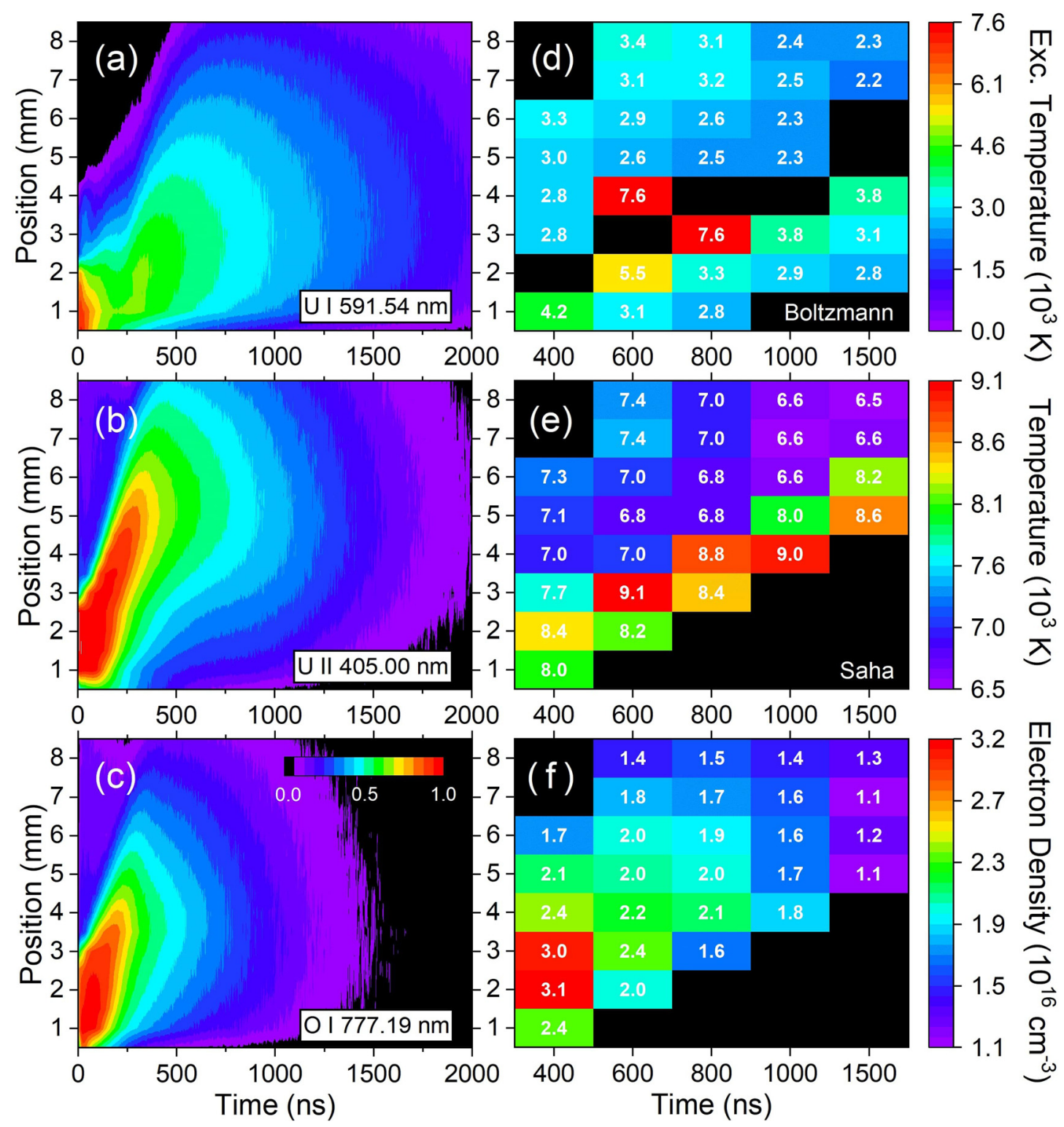

FIG. 4. (a) U I $591.538 \mathrm{~nm}$, (b) U || $405.00 \mathrm{~nm}$, and (c) O I $777.19 \mathrm{~nm}$ spatiotemporally resolved emission. Temperature distribution obtained via (d) Boltzmann s and (e) the Saha-Eggert equation and (f) electron density in the uranium LIP at various time delays and axial positions with respect to the target surface. The absence of values along the diagonal in (d) is due to inability to clearly observe the majority of spectral lines at the corresponding positions and time delays.

\section{B. Emission spectra}

At an early time delay, the LIP emission is dominated by continuum radiation. The emission diminishes significantly at $1000 \mathrm{~ns}$ time delay at all axial positions. At this point, the LIP decay is accompanied by the reduction of spectral line broadening to the minimum defined by an instrumental FWHM. A comparison between the simulated atomic spectrum at late time delay and the measured spectrum is shown in Fig. 5. The simulation was performed for U I transitions in the given spectral region with a temperature $5000 \mathrm{~K}$. The modeled spectrum accounts for the majority of the U I and U II lines observed in the experimental spectrum.

\section{Plasma diagnostics}

\section{Excitation and electron temperatures}

Plasma excitation temperature calculations were performed using the Boltzmann plot method, which estimates the temperature based on the intensity ratios of spectral lines. Using the relation

$$
\ln \left(\frac{I_{k i} \lambda}{g_{k} A_{k i}}\right)=-\frac{E_{k}}{k_{B} T_{e x c}}+\ln \left(\frac{\hbar c N_{e}}{2 U\left(T_{e x c}\right)}\right),
$$

the excitation temperature can be determined from the slope of the left-hand side term vs $E_{k}$, where $I_{k i}$ is the intensity of the transition, $\lambda$ 


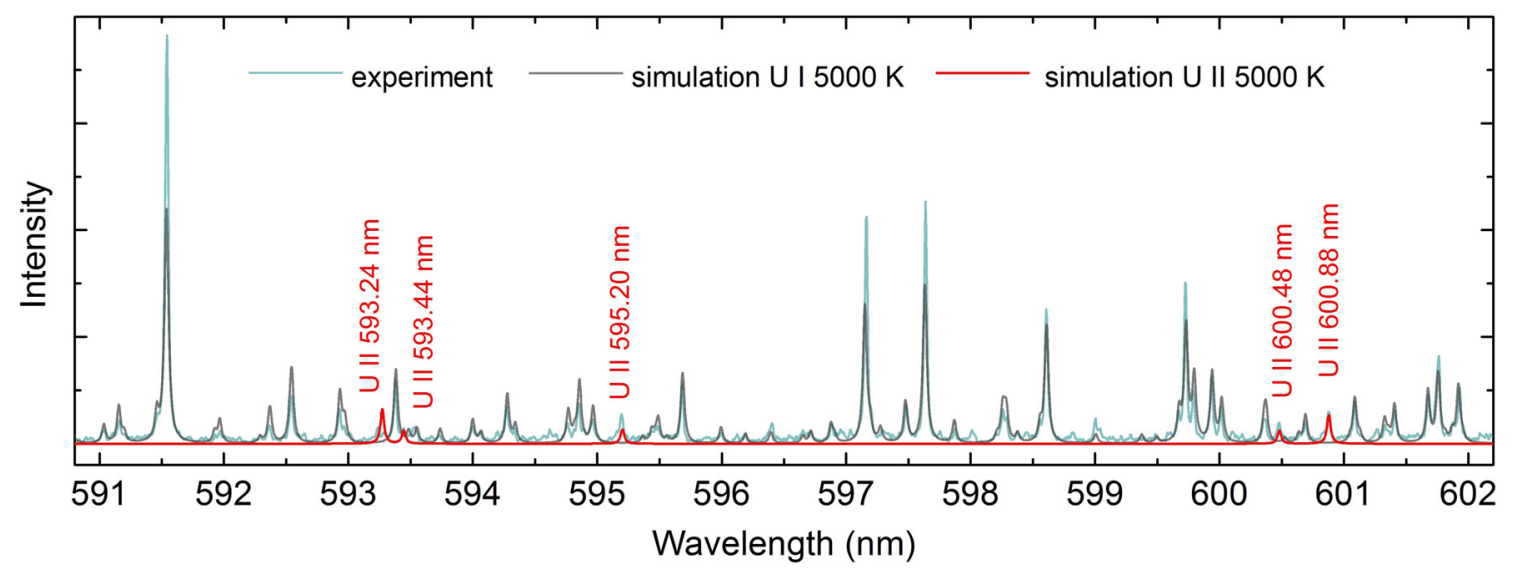

FIG. 5. Generated spectrum for $5000 \mathrm{~K}$ and experimental spectrum recorded at $1000 \mathrm{~ns}$ and $2 \mathrm{~mm}$ away from the target.

is the wavelength of the transition, $g_{k}$ is the degeneracy of the upper state, $A_{k i}$ is the transition probability, $k_{B}$ is the Boltzmann constant, $T_{\text {exc }}$ is the excitation temperature, $E_{k}$ is the energy of the upper state, $\hbar$ is the reduced Planck constant, $c$ is the speed of light, and $U\left(T_{\text {exc }}\right)$ is the partition function. Subscripts $i$ and $k$ represent the excitation states for the transitions. The validity of this method for temperature estimates hinges on the assumption of local thermal equilibrium (LTE) within the plume. Only the lines of atomic uranium are considered. The choice of lines is such to avoid resonance transitions or strong spectral lines which can be prone to the self-absorption effect. All lines that were used for the construction of Boltzmann plots are listed in Table I, with the atomic parameters obtained from the Palmer Spectral Atlas. ${ }^{19}$ The intensity of the lines is approximated by the area underneath the Voigt profile. Figure 4(d) shows the spatiotemporal excitation temperature distribution; the temperature on the plasma periphery is significantly lower than that in the core. The typical Boltzmann plots for two different time delays are shown in Fig. 6. Errors of the spectral line intensity estimation vary from 5 to $17 \%$. The Boltzmann plot data points lying outside of this range (typically corresponding to the lines centered at $421.16,454.36,602.81$, and $651.89 \mathrm{~nm}$ ) were treated as outliers and were not considered in further analysis.

The validity of LTE conditions in high-Z plasma can be verified by comparing the excitation and electron temperatures. The plasma electron temperature was calculated using the Saha-Eggert equation, ${ }^{37}$ relying on the intensity ratios of several neutral and single-ionized $U$ spectral lines

$$
\begin{aligned}
\frac{I_{n m}^{j+1}}{I_{k i}^{j}} N_{e}= & \frac{A_{n m}^{j+1} g_{n}^{j+1} \lambda^{j+1}}{A_{k i}^{j} g_{k}^{j} \lambda^{j}}\left(\frac{2 \pi m_{e} k_{B} T_{e}}{h^{2}}\right)^{3 / 2} \\
& \times \exp \frac{-\left(E_{n}^{j+1}-E_{k}^{j}+\Delta E^{j+1, j}\right)}{k_{B} T_{e}},
\end{aligned}
$$

where $\Delta E^{j+1, j}$ represents the ionization potential between ionization states $j$ and $j+1 ; m_{e}$ and $T_{e}$ are the electron mass and temperature, respectively; and $h$ is the Planck constant. Subscripts $i, k, m$, and $n$ represent the excitation states associated with the transitions. Table II contains the three atomic and four ionic $\mathrm{U}$ spectral lines used in the analysis, yielding twelve total line pair combinations. Lines were strategically chosen to avoid significant overlap with adjacent features and self-absorption by excluding resonance transitions. The Saha-Eggert equation was solved iteratively for each of the twelve combinations using the electron densities determined via the analysis of Stark broadening of the oxygen $777.19 \mathrm{~nm}$ line discussed in Sec. IV C2. Figure 4 (e) contains the average of the electron temperatures determined from the twelve combinations. The standard deviation of the twelve measurements ranged from $10 \%$ to $15 \%$ at different spatiotemporal positions. The electron temperatures obtained with this method are significantly higher than those obtained by the Boltzmann plot method. This difference is even more pronounced at the plasma periphery.

\section{Electron density}

Since the electron density at early time delays is large, it makes a significant contribution to line broadening. Due to the lack of Stark broadening parameters for $U$ lines, we used the neutral oxygen surface impurity line at $777.19 \mathrm{~nm}$ to infer the plasma electron density in the early stages of plasma evolution. Our PMT measurements suggest that the majority of the oxygen impurity in the LIP originates from the target. The atomic oxygen line peak centered at $777.19 \mathrm{~nm}$ is spectrally resolved with respect to the rest of the lines within the triplet (777.19, 777.42 , and $777.54 \mathrm{~nm}$ ). We consider three primary mechanisms contributing to the broadening of the spectral lines: Stark, Doppler, and instrumental broadening. Since oxygen triplet lines are not resonance lines, the resonance broadening is not considered. For ablation in vacuum or low pressure, where ablated species exhibit high expansion velocities, Doppler broadening needs consideration. The spectral line shape associated with Doppler broadening corresponds to the Gaussian function. The Doppler FWHM for oxygen spectral lines $\left(\Delta \lambda_{D}=7.2 \times 10^{-7} \lambda_{0} \sqrt{T_{e} / M}\right.$, with $\lambda_{0}$ being the center wavelength and $M$ being the molar mass) was found to be $14 \mathrm{pm}$ at $10000 \mathrm{~K}$ and $10 \mathrm{pm}$ at $5000 \mathrm{~K}$. By setting the spectrograph at its maximum resolution, one can minimize the instrumental broadening. Stark broadening is caused by the fast varying local electric fields generated by the free charged particles of the plasma, whereas the Stark shift is attributed to the slowly varying electric fields as the noncoherent superposition of spectral lines individually shifted by the Stark effect. ${ }^{38,39}$ The FWHM of a Stark-broadened line is given by ${ }^{40}$ 
TABLE I. Atomic parameters ${ }^{19}$ of $\mathrm{U}$ I spectral lines considered for the determination of excitation temperature.

\begin{tabular}{|c|c|c|c|c|c|}
\hline$\lambda(\mathrm{nm})$ & $E_{u}(\mathrm{eV})$ & $J_{u}$ & $E_{l}(\mathrm{eV})$ & $J_{l}$ & $g f$ \\
\hline 405.81 & 3.52 & 7 & 0.47 & 7 & $1.29 \times 10^{-9}$ \\
\hline 410.31 & 3.93 & 8 & 0.91 & 7 & $8.40 \times 10^{-10}$ \\
\hline 416.24 & 3.45 & 8 & 0.47 & 7 & $1.48 \times 10^{-9}$ \\
\hline 416.37 & 3.51 & 7 & 0.53 & 6 & $9.67 \times 10^{-10}$ \\
\hline 418.70 & 3.49 & 7 & 0.53 & 6 & $1.24 \times 10^{-9}$ \\
\hline 420.11 & 3.90 & 9 & 0.95 & 8 & $7.57 \times 10^{-10}$ \\
\hline 421.16 & 3.72 & 6 & 0.77 & 6 & $1.03 \times 10^{-9}$ \\
\hline 422.24 & 3.41 & 8 & 0.47 & 7 & $2.62 \times 10^{-9}$ \\
\hline 423.17 & 3.88 & 9 & 0.95 & 8 & $1.01 \times 10^{-9}$ \\
\hline 426.79 & 3.68 & 6 & 0.77 & 6 & $7.72 \times 10^{-10}$ \\
\hline 428.88 & 3.66 & 6 & 0.77 & 6 & $1.61 \times 10^{-9}$ \\
\hline 431.31 & 3.34 & 8 & 0.47 & 7 & $2.11 \times 10^{-9}$ \\
\hline 436.28 & 3.31 & 8 & 0.47 & 7 & $9.14 \times 10^{-10}$ \\
\hline 437.18 & 3.84 & 8 & 1.01 & 7 & $1.09 \times 10^{-9}$ \\
\hline 444.83 & 3.26 & 7 & 0.47 & 7 & $7.92 \times 10^{-10}$ \\
\hline 462.02 & 3.46 & 7 & 0.77 & 6 & $2.59 \times 10^{-9}$ \\
\hline 474.35 & 3.39 & 7 & 0.77 & 6 & $1.27 \times 10^{-9}$ \\
\hline 481.09 & 3.05 & 8 & 0.47 & 7 & $1.97 \times 10^{-9}$ \\
\hline 481.57 & 3.04 & 7 & 0.47 & 7 & $1.08 \times 10^{-9}$ \\
\hline 491.04 & 3.30 & 7 & 0.77 & 6 & $2.13 \times 10^{-9}$ \\
\hline 492.84 & 3.04 & 7 & 0.53 & 6 & $3.02 \times 10^{-9}$ \\
\hline 495.58 & 3.45 & 8 & 0.95 & 8 & $1.33 \times 10^{-9}$ \\
\hline 496.73 & 2.97 & 8 & 0.47 & 7 & $2.53 \times 10^{-9}$ \\
\hline 506.38 & 2.92 & 7 & 0.47 & 7 & $1.58 \times 10^{-9}$ \\
\hline 516.41 & 3.41 & 8 & 1.01 & 7 & $1.53 \times 10^{-9}$ \\
\hline 530.85 & 2.81 & 7 & 0.47 & 7 & $3.61 \times 10^{-9}$ \\
\hline 549.64 & 2.76 & 6 & 0.53 & 6 & $1.95 \times 10^{-9}$ \\
\hline 556.42 & 2.70 & 7 & 0.47 & 7 & $6.68 \times 10^{-9}$ \\
\hline 561.09 & 2.98 & 7 & 0.77 & 6 & $2.88 \times 10^{-9}$ \\
\hline 562.15 & 2.68 & 6 & 0.47 & 7 & $2.23 \times 10^{-9}$ \\
\hline 578.06 & 2.92 & 7 & 0.77 & 6 & $4.04 \times 10^{-9}$ \\
\hline 597.63 & 2.55 & 8 & 0.47 & 7 & $1.02 \times 10^{-8}$ \\
\hline 598.61 & 2.55 & 4 & 0.48 & 3 & $4.71 \times 10^{-9}$ \\
\hline 599.73 & 2.84 & 7 & 0.77 & 6 & $3.87 \times 10^{-9}$ \\
\hline 602.81 & 2.54 & 2 & 0.48 & 3 & $1.98 \times 10^{-9}$ \\
\hline 606.23 & 2.57 & 7 & 0.53 & 6 & $2.11 \times 10^{-9}$ \\
\hline 617.19 & 2.96 & 9 & 0.95 & 8 & $4.13 \times 10^{-9}$ \\
\hline 617.54 & 2.54 & 7 & 0.53 & 6 & $4.27 \times 10^{-9}$ \\
\hline 621.54 & 2.46 & 7 & 0.47 & 7 & $2.76 \times 10^{-9}$ \\
\hline 629.85 & 2.50 & 5 & 0.53 & 6 & $2.96 \times 10^{-9}$ \\
\hline 637.25 & 2.42 & 8 & 0.47 & 7 & $9.95 \times 10^{-9}$ \\
\hline 638.98 & 2.68 & 5 & 0.74 & 4 & $3.87 \times 10^{-9}$ \\
\hline 646.50 & 2.82 & 8 & 0.91 & 7 & $7.05 \times 10^{-9}$ \\
\hline 650.36 & 2.44 & 7 & 0.53 & 6 & $3.88 \times 10^{-9}$ \\
\hline 651.89 & 2.68 & 6 & 0.77 & 6 & $3.02 \times 10^{-9}$ \\
\hline 655.50 & 2.37 & 2 & 0.48 & 3 & $4.34 \times 10^{-9}$ \\
\hline
\end{tabular}

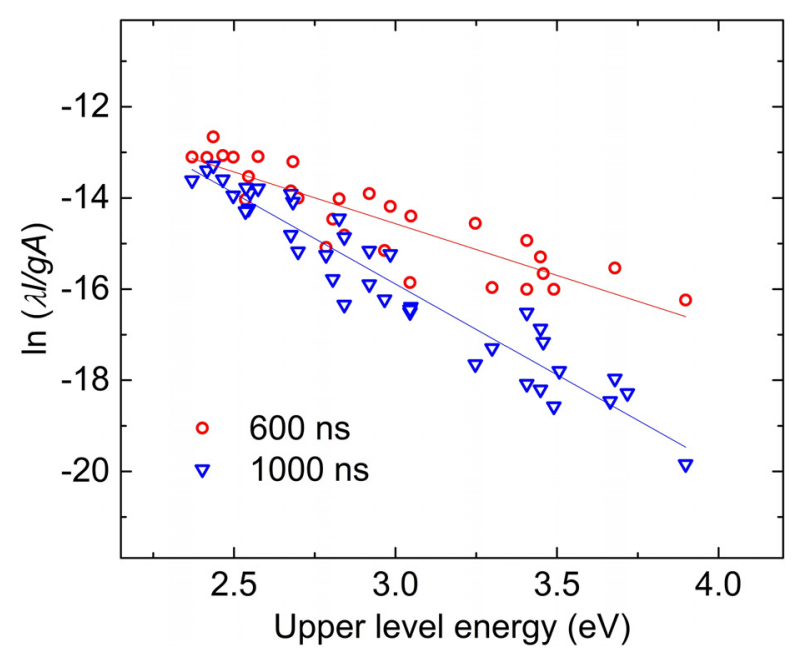

FIG. 6. Boltzmann plots obtained at 2-mm axial positions at two different time delays. The $U$ transition probabilities were taken from the Palmer Spectral Atlas.

$$
\Delta \lambda_{S}=2 W\left(\frac{N_{e}}{10^{16}}\right)
$$

where $W$ is the electron-impact parameter. It should be noted that Eq. (3) does not include the ionic contribution. The correction for nonhydrogenic ion Stark broadening is

$$
\Delta \lambda_{i}=3.5 A\left(\frac{N_{e}}{10^{16}}\right)^{5 / 4}\left(1-\frac{3}{4} N_{D}^{-1 / 3}\right) W,
$$

where $A$ is the static ion broadening parameter and $N_{D}$ is the number of particles within the Debye sphere. Under our experimental conditions, ionic contribution was found to be negligible. The electronimpact parameter of $16.6 \mathrm{pm}$ is obtained from the literature ${ }^{41}$ for the temperature of $\sim 0.5 \mathrm{eV}$. The errors in the impact parameters were estimated to be $\pm 20 \%$. We estimated the density of the plasma by assuming that the impact parameters would vary negligibly in the temperature range from 5000 to $10000 \mathrm{~K}$. Figure 4(f) shows the spatiotemporal electron density distribution. Typical Stark-broadened profiles of oxygen triplet lines are given in Fig. 7. The experimental curves shown fit reasonably well with a convolution of three Voigt profiles. We applied the standard deconvolution procedure based on the least squares fitting described in detail elsewhere. ${ }^{42}$

TABLE II. Atomic parameters ${ }^{19}$ of $\mathrm{U} I$ and $\mathrm{U}$ II spectral lines used for the determination of electron temperature via the Saha-Eggert equation.

\begin{tabular}{lccccc}
\hline \hline Species & $\lambda(\mathrm{nm})$ & $E_{u}(\mathrm{eV})$ & $J_{u}$ & $E_{l}(\mathrm{eV})$ & $J_{l}$ \\
\hline U I & 530.85 & 2.81 & 7 & 0.47 & 7 \\
U I & 578.06 & 2.92 & 7 & 0.77 & 6 \\
U I & 597.63 & 2.55 & 8 & 0.47 & 7 \\
U II & 417.16 & 3.19 & 6.5 & 0.12 & 6.5 \\
U II & 434.17 & 2.89 & 4.5 & 0.07 & 5.5 \\
U II & 447.23 & 2.81 & 4.5 & 0.06 & 5.5 \\
U II & 500.82 & 2.69 & 6.5 & 0.02 & 6.5 \\
\hline
\end{tabular}




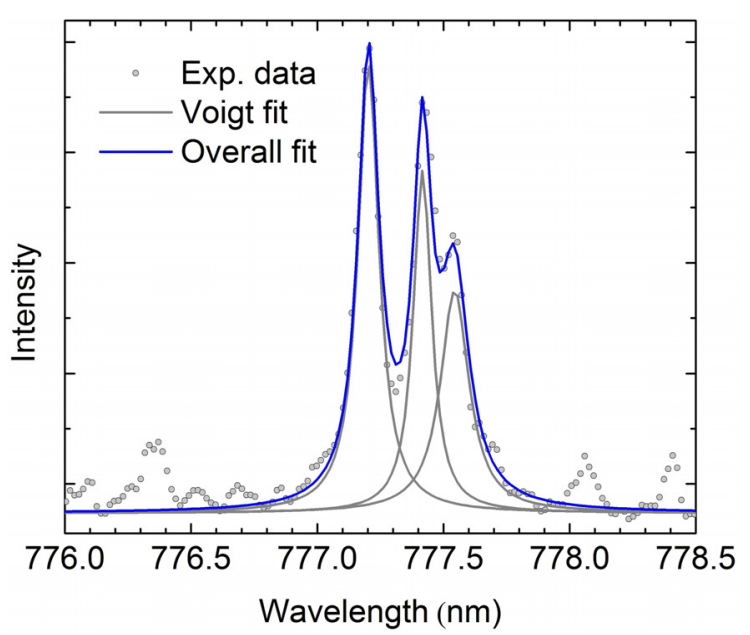

FIG. 7. Typical profiles of oxygen lines at $777.19,777.42$, and $777.54 \mathrm{~nm}$ recorded under low pressure [0.67 Pa $(5 \mathrm{mTorr})]$. The cumulative Voigt fit is used to model the triplet structure.

\section{Determination of Stark widths}

The difficulty in asserting the optimal conditions for the deduction of Stark widths is a nontrivial task in the case of highly congested spectrum such as the one from uranium. There are several criteria that need to be met in order to choose a suitable candidate spectral line. This line should be (i) isolated, (ii) sufficiently broadened, (iii) intense, and (iv) optically thin. However, the latter two are the opposed requirements, which may a priori exclude resonant lines. Indeed, the most intense spectral lines in the uranium spectra are resonant, including both atomic and ionic transitions. Most of these lines are merged with the ones that are less intense or closely spaced, even in a low pressure environment and reduced continuum radiation. Under these circumstances, we have found one atomic and one ionic spectral line which meet the above-mentioned criteria. The fitting procedure for uranium lines was analogous to the one used for oxygen lines. The Gaussian width due to the Doppler broadening and Gaussian fraction of the instrumental profile was used as a fixed parameter during Voigt fitting. After obtaining Lorentzian FWHM from the Voigt fit, we subtracted the Lorentzian instrumental FWHM to finally obtain Stark FWHM. Doppler broadening for U I and U II transitions at a temperature of $10000 \mathrm{~K}$ is calculated to be $2.3 \mathrm{pm}$. Figure 8 shows the electron density dependence of U I $499.01 \mathrm{~nm}$ and U II $500.82 \mathrm{~nm}$ spectral line Stark widths measured for the spectra recorded with different time delays. Figure 9 shows the temporal evolution of U I $499.01 \mathrm{~nm}$ and U II $500.82 \mathrm{~nm}$ line profiles recorded $1.5 \mathrm{~mm}$ away from the target surface. The line profiles of U I $499.01 \mathrm{~nm}$ and II $500.82 \mathrm{~nm}$ fitted to the Voigt function are shown in Figs. 10(a) and 10(b), respectively. We note that the U line intensities estimated by the free-parameter Voigt fit corresponded to the values obtained employing the Lorentzian function within $\sim 2 \%$ in average. The Stark width is determined by the slope of the linear fit shown in Fig. 8 according to Eq. (3). The results of the Stark width estimations normalized to $1 \times 10^{16} \mathrm{~cm}^{-3}$ are presented in Table III.

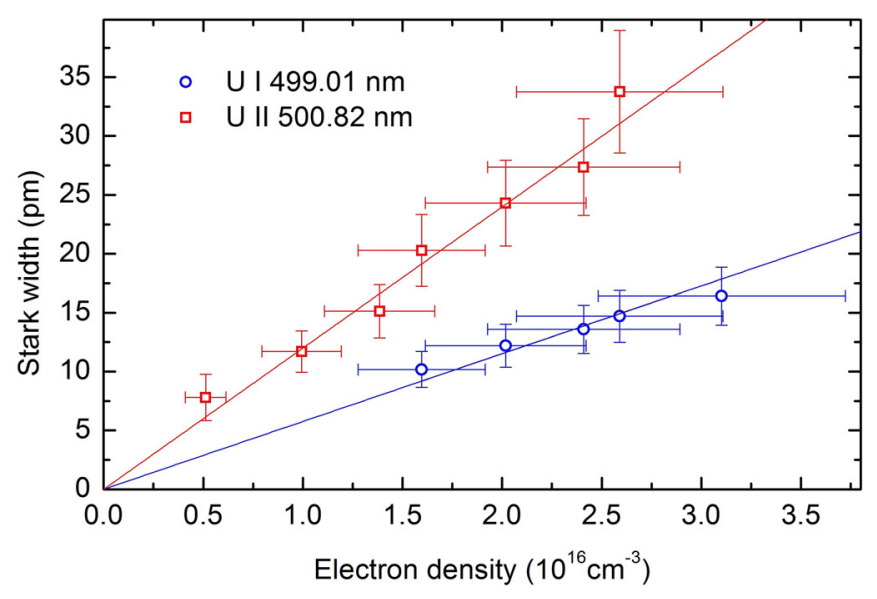

FIG. 8. Dependence of Stark widths of U I| $500.82 \mathrm{~nm}$ and U | $499.01 \mathrm{~nm}$ on electron density.

\section{DISCUSSION}

The characteristic cylindrical shape of the LIP in the lowpressure environment is evidenced by fast-gated plume imaging employing the ICCD (Fig. 2) at time delays larger than $200 \mathrm{~ns}$ and 4 times faster expansion in the axial direction. The fact that the expansion occurs undisturbed in the low pressure conditions and the relatively large focusing spot diameter determined the overall plasma geometry. Previous work documented the changes in the plume morphology with varying spot sizes, observing a transition between spherical and cylindrical expansions by changing the focal spot diameter from 60 to $280 \mu \mathrm{m} .{ }^{43}$ The spatiotemporal contours in Fig. 4 show that ionic species expand faster than atomic species in the plasma. The higher velocity of ionic species is attributed to the space-charge effect. ${ }^{44}$ Although the role of the ambient oxygen contribution should not be entirely ruled out, the similar KE (Fig. 3) between atomic $\mathrm{U}$ and

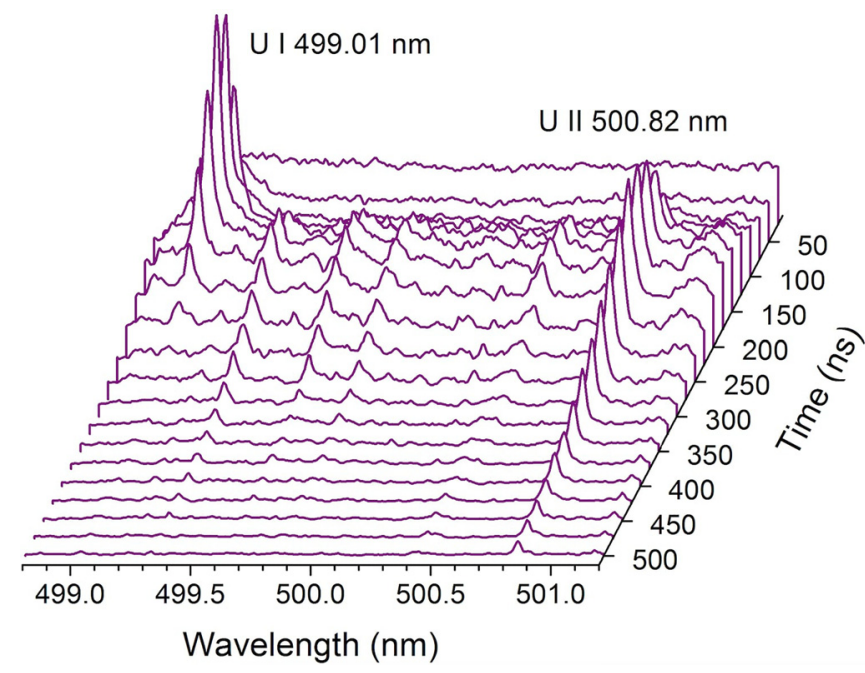

FIG. 9. Temporal evolution of $U$ | 499.01-nm and U I| 500.82-nm line profiles recorded $1.5 \mathrm{~mm}$ away from the target surface. 

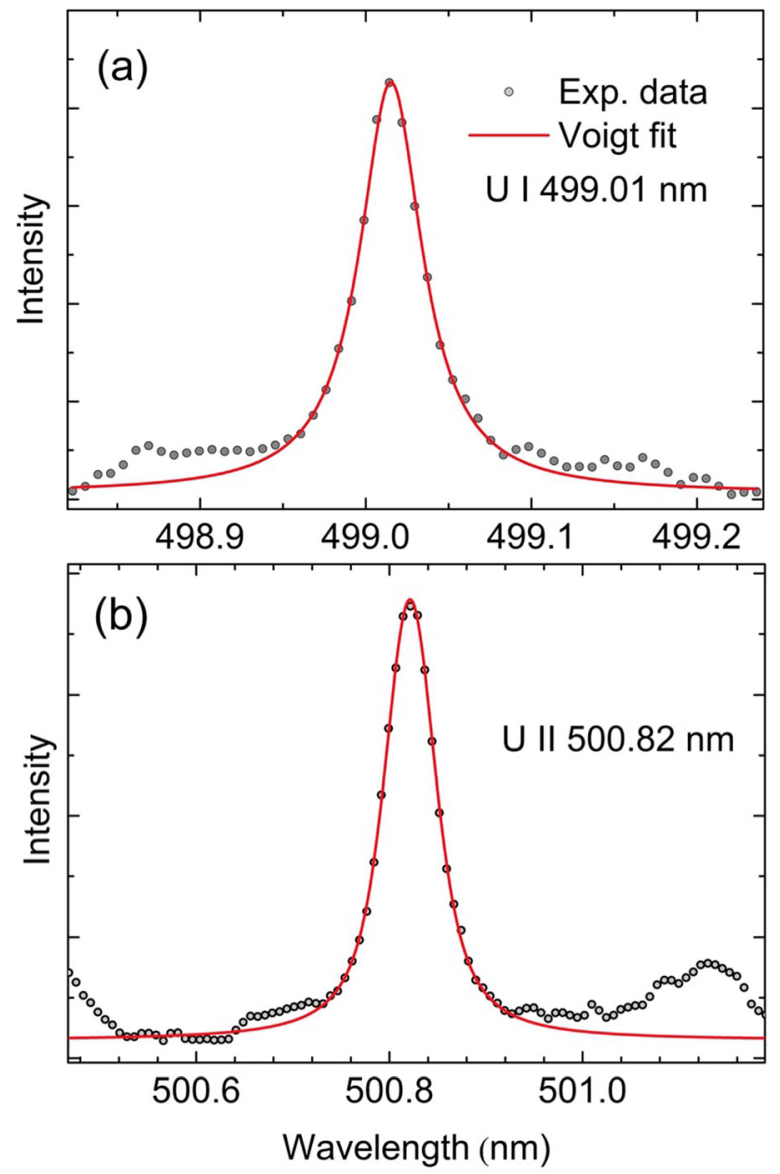

FIG. 10. Typical profiles of (a) U I 499.01-nm and (b) U I| 500.82-nm spectral lines fitted to the Voigt function.

O species supports the conjecture that the $\mathrm{O}$ impurity originates primarily from the target. This is further verified by the spatiotemporal shape of emission contours in the LIP [Figs. 4(a)-(c)].

The persistence of any species in the LIP depends on plasma thermodynamic parameters, its local number density in the plume, and the upper level energy and characteristic lifetime for the particular transition. Figure 5 reveals highly congested spectral features in the LIP as a consequence of densely packed upper energy levels of both U I and U II. The simulated spectrum (Fig. 5) showed most of the lines seen in the experimental spectrum. The disagreement in intensities of various transitions in the experiment with respect to simulation can be correlated with the line-averaged measurement and nonhomogeneous

TABLE III. Atomic parameters ${ }^{19}$ of U I $(499.01 \mathrm{~nm})$ and U II $(500.82 \mathrm{~nm})$ spectral lines together with the measured Stark widths normalized to $10^{16} \mathrm{~cm}^{-3}$.

\begin{tabular}{lcccccc}
\hline \hline Species & $\lambda(\mathrm{nm})$ & $E_{u}(\mathrm{eV})$ & $J_{u}$ & $E_{l}(\mathrm{eV})$ & $J_{l}$ & $\lambda_{S}(\mathrm{pm})$ \\
\hline U I & 499.01 & 3.49 & 7 & 1.01 & 7 & 6 \\
U II & 500.82 & 2.69 & 6.5 & 0.22 & 6.5 & 12 \\
\hline \hline
\end{tabular}

plasma, the lack of radiation transport effects in the model, and the accuracy of the relative oscillator strengths.

Due to its pyrophoric character, U oxidizes very rapidly by interacting with ambient oxygen even at vacuum-like conditions. In the present experiments, since we use $0.67 \mathrm{~Pa}$ ( $5 \mathrm{mTorr}$ ) ambient pressure, the oxidation of the target cannot be ruled out. The time required for one monolayer formation in the vacuum level we used is $\sim 300 \mu \mathrm{s}$ assuming the unity sticking coefficient. ${ }^{45}$ Therefore, approximately 300 oxide monolayers can be formed between laser pulses (100 ms).

Measurements of temperature and electron density rely on averaging the emission of spectral lines along the line of sight. In addition, many lines could not be distinguished when observing plasma regions where the highest electron densities and temperatures are expected. The absence of values at early time delays and short distances from the target observed in Fig. 4(d) reflect the inability to determine the excitation temperature via Boltzmann plots under given conditions. Interestingly, the spatiotemporal locations at which we were unable to use the Boltzmann plot to determine the excitation temperature coincide with the highest electron temperature locations obtained via the Saha-Eggert equation [Fig. 4(e)]. We observed weak emission from atomic species in these locations caused by a high ionization fraction at these positions.

The differences in excitation temperatures obtained via the Boltzmann plot method and electron temperatures determined through the Saha-Eggert equation imply a departure from LTE. In the case of LTE, the excitation and electron temperatures should be equal. The LTE condition implies that collisional processes, rather than radiative processes, are the dominant form of deexcitation and energy loss in the plasma and that the temperature of the system determines the relative populations of excited levels of a species. This is referred to as the Atomic State Distribution Function and is described by the Boltzmann distribution. ${ }^{40}$ We have considered the plasma to be in LTE in the estimation of temperature from spectral data. The issue of LTE in laser-produced plasmas has been discussed by several authors. $^{37,40,46}$ In a transient system, such as a plasma formed by a pulsed laser source, the existence of LTE requires that the electronatom and electron-ion collisional processes occur sufficiently fast and dominate (an order of magnitude) over radiative processes. In a system that is in LTE, particles will have Maxwellian velocity distributions, population levels will be distributed according to Boltzmann statistics, ionization states will be described by the Saha-Eggert equation, and the radiation density will follow the Planck law.

The McWhirter criterion ${ }^{23}$ is often used to verify LTE conditions and justify temperature estimates using the Boltzmann plot method by establishing a minimum electron number density required to assume LTE. While this criterion can be fulfilled at a sufficient delay after the laser pulse, it is not sufficient to verify LTE because it is clear that the inhomogeneous, transient nature of LIBS plasmas does not fully satisfy LTE conditions, and in these cases, results must be considered with precaution. ${ }^{46}$ In the present experiment where the plume expands freely into a low pressure environment, the plume species move with different expansion velocities, and the efficiency of three-body recombination will be reduced farther from the target. It is known that species with higher charge states propagate much faster than their atomic counterparts and neutrals in general as supported in Figs. 4(a)-4(c). The reason appears to be that equilibration between excited electronic levels and the ground state is slower than the equilibration between 
the translational energies of the free electrons and heavy particles. Therefore, at larger distances where the differential expansion becomes noticeable, the various species will become segregated which in turn reduces the collision frequency. This will reduce the rate of temperature equilibration, potentially contravening the assumption of local thermodynamic equilibrium in the plasma. In the present experiment, we assume that the central (core) region of the plume may be close to the LTE or at least partial LTE (pLTE) conditions. The Boltzmann plots, used for estimating the temperature from line intensities of various $\mathrm{U}$ lines, showed a straight line fit (Fig. 6). However, a straight line may be obtained in the Boltzmann plot even when non-pLTE levels are included; thus, this method may lead to obtaining spuriously low excitation temperatures. Another plausible explanation for the differences between excitation and electron temperatures is differing spatial distributions of various plasma species. Previous work demonstrates the effects of considering spatial distributions of species by comparing the line-of-sight averaged spectral data with the Abel inverted data which account for radial symmetry in the plume. ${ }^{47}$ Therefore, the temperatures reported in this work should be considered with caution since line-of-sight averaging was not accounted for.

Due to the uncertainties in transition probabilities of at least $\sim 20 \%{ }^{19}$ and difficulties associated with evaluating the intensities of congested spectral lines, there is a relatively large scatter of the data points, resulting with error in the $T_{\text {exc }}$ estimation of about $35 \%$. On the other hand, temperatures obtained with the Saha-Eggert equation have an error of up to $\sim 20 \%$. The final error in the electron density estimation of about $25 \%$ is dictated by the errors in the Stark broadening from the literature ${ }^{41}$ Furthermore, we confirm the validity of the McWhirter criterion $^{23}$ in the present experiment, which states that the minimum electron density for LTE should be $N_{e} \geq 1.6 \times 10^{12} T_{e x c}^{1 / 2}(\Delta E)^{3}$, where $\Delta E$ is the largest gap between adjacent upper energy levels. For the largest energy gap of $\Delta E \sim 2 \mathrm{eV}$, applying the peak plume temperature of $8400 \mathrm{~K}$ to this criterion predicts a lower limit for $N_{e}$ of $1 \times 10^{15} \mathrm{~cm}^{-3}$. Our observed values of $N_{e}$ are always greater than this lower bound up to about an order of magnitude, implying that the LTE approximation assumed for our analysis is valid. These are necessary conditions for LTE but not sufficient. At larger distances and greater time delays, the number densities of the plasma are low, and the boundary layer moves rapidly, undermining the LTE assumption. This is supported by the fact that the spatially integrated electron density dropped at the plasma borders to $1 \times 10^{16} \mathrm{~cm}^{-3}$ which is considerably lower than the measured initial density values obtained from time-resolved studies. The drop in density will affect the thermal equilibrium established between the upper levels of uranium atomic transitions used for the Boltzmann plot. It has been shown the LIP is hotter and denser at a closer distance to the ablating target and at early time delays when the Stark effect is predominant. ${ }^{26}$ Therefore, we can conclude that LTE may be assumed for the core region and early time delays and also as an inadequate approximation for large distances and late stages of plasma evolution when it expands in vacuum or low-pressure conditions. A recent comparison study ${ }^{17}$ involving the diagnostics of uranium LIP in Ar and air shows temperature drop from $12000 \mathrm{~K}$ to $4000 \mathrm{~K}$ within $20 \mu$ s. Interestingly, the authors showed a similar emission persistence time of ionic and atomic spectral lines for both environments. It would be reasonable to assume that the persistence of these transitions would also be comparable under low pressures. Indeed, the upper energy levels for U II transitions are not high lying with respect to the ones from U I but rather at similar values. This applies to the corresponding oscillator strengths for U II transitions as well. Similar persistence times for U I and U II species can be observed in Figs. 4(a) and 4(b).

In order to estimate the Stark width, it is often helpful to consider the overall trend in Stark width behavior with electron density rather than relying on one particular spectrum. This is especially important when the electron density values are low, signifying the fading of the Stark broadening contribution (Fig. 8). The main source of error in Stark width estimation for U I and U II transitions is associated with error in electron density estimation via the O I $777.19 \mathrm{~nm}$ line. According to the linear increase observed in Fig. 8, the relative errors arising from the linear analysis are small compared to the absolute errors associated with the electron density measurement using the $\mathrm{O} I$ 777.19-nm spectral line. However, considering that the $N_{e}$-range in our experiments was restricted by experimental conditions which allowed the observation of U I 499.01-nm and II 500.82-nm lines, the measurement errors are naturally larger. This is accompanied by errors in the determination of instrumental broadening and by the error in the fitting procedure. Figure 10 shows late time delay profiles where the FWHM of both lines does not exceed $\sim 100 \mathrm{pm}$. Consequently, the error is relatively large when the instrumental FWHM is comparable to the overall FWHM. ${ }^{48}$ We estimate the error of Stark width determination to be within a tolerable margin of $\sim 45 \%$. Due to the lack of the existing data in the literature, we are unable to perform the direct comparison of our measured Stark width values. Nevertheless, the order of magnitude of values reported here is consistent with typical values in the existing literature spanning from several tens to several hundreds of $\mathrm{pm}$ at $10^{17} \mathrm{~cm}^{-3}$.

\section{CONCLUSION}

Optical emission spectroscopy is used to characterize the laserproduced plasma from uranium under low pressure conditions. At earlier time delays, intense continuum radiation is dominant, making it difficult to extract line intensities and profiles. We analyze the optical emission at later delays and at various distances from the target in order to determine space- and time-dependent plasma properties including excitation and electron temperatures and electron density. Line intensities from the atomic $U$ species were used in the Boltzmann plot method for determining excitation temperatures. We compare excitation temperatures obtained using the Boltzmann plots to electron temperatures from the Saha-Eggert equation. Measurements of electron density were conducted using a surface impurity oxygen line O I $777.19 \mathrm{~nm}$. In the early stage of plasma evolution, we find the temperature and density rapidly decrease, which is characteristic of an adiabatic expansion. The electron density is found to decay more slowly in the plasma periphery at greater distances from the target. Space- and time-resolved measurements of excitation and electron temperatures reveal a greater difference between the two values in the plasma periphery. We discuss the existence of pLTE in the periphery evidenced by the differing excitation and electron temperatures while still satisfying the McWhirter criterion. Another source of the discrepancy in temperatures may arise from the line of sight measurements. ${ }^{47}$ In order to address this question, our future work will involve radially resolved analysis via Abel inversion.

The spatiotemporal region corresponding to the higher temperature plasma core was considered for Stark broadening measurements. Thus, the low pressure environment, although not ideal, provided a compromise between excessive spectral overlapping between 
congested uranium spectral lines and the measurable degree of Stark broadening. Under these conditions, we performed the measurements of the Stark width for U I 499.01-nm and U II 500.82-nm lines. These measurements present a step toward comprehensive modeling of $U$ emission spectra and a reference for further investigations of U Stark coefficients.

\section{ACKNOWLEDGMENTS}

This work was supported by the Department of Energy (DOE), National Nuclear Security Administration (NNSA), Consortium for Verification Technology (CVT) (No. DENA0002534); the Office of Defense Nuclear Nonproliferation (DNN) (No. NA-22); and the National Science Foundation Graduate Research Fellowships Program (NSF GRFP) (No. DGE 1256260). Pacific Northwest National Laboratory (PNNL) is operated for the U.S. DOE by the Battelle Memorial Institute (No. DE-AC05-76RLO1830), PNNL Radiological Services Department.

\section{REFERENCES}

${ }^{\mathbf{1}}$ W. Pietsch, A. Petit, and A. Briand, Spectrochim. Acta, Part B 53, 751 (1998).

${ }^{2}$ R. C. Chinni, D. A. Cremers, L. J. Radziemski, M. Bostian, and C. Navarro-

Northrup, Appl. Spectrosc. 63, 1238 (2009).

${ }^{3}$ A. Sarkar, D. Alamelu, and S. K. Aggarwal, Talanta 78, 800 (2009).

${ }^{4}$ F. R. Doucet, G. Lithgow, R. Kosierb, P. Bouchard, and M. Sabsabi, J. Anal. At. Spectrom. 26, 536 (2011).

${ }^{5}$ L. A. Emmert, R. C. Chinni, D. A. Cremers, C. R. Jones, and W. Rudolph, Appl. Opt. 50, 313 (2011).

${ }^{6}$ D. A. Cremers, A. Beddingfield, R. Smithwick, R. C. Chinni, C. R. Jones, B. Beardsley, and L. Karch, Appl. Spectrosc. 66, 250 (2012).

${ }^{7}$ N. R. Taylor and M. C. Phillips, Opt. Lett. 39, 594 (2014).

${ }^{8}$ G. C.-Y. Chan, I. Choi, X. Mao, V. Zorba, O. P. Lam, D. K. Shuh, and R. E. Russo, Spectrochim. Acta, Part B 122, 31 (2016).

${ }^{9}$ K. C. Hartig, I. Ghebregziabher, and I. Jovanovic, Sci. Rep. 7, 43852 (2017).

${ }^{10}$ X. Mao, G. C.-Y. Chan, I. Choi, V. Zorba, and R. E. Russo, J. Radioanal. Nucl. Chem. 312, 121 (2017).

${ }^{11}$ D. G. Weisz, J. C. Crowhurst, W. J. Siekhaus, T. P. Rose, B. Koroglu, H. B. Radousky, J. M. Zaug, M. R. Armstrong, B. H. Isselhardt, M. R. Savina, M. Azer, M. S. Finko, and D. Curreli, Appl. Phys. Lett. 111, 034101 (2017).

${ }^{12}$ S. S. Harilal, B. E. Brumfield, N. L. LaHaye, K. C. Hartig, and M. C. Phillips, Appl. Phys. Rev. 5, 021301 (2018).

${ }^{13}$ D. A. Cremers and L. J. Radziemski, Handbook of Laser-Induced Breakdown Spectroscopy (John Wiley \& Sons, NJ, 2013).

${ }^{14}$ J. Hermann, D. Grojo, E. Axente, C. Gerhard, M. Burger, and V. Craciun, Phys. Rev. E 96, 053210 (2017).

${ }^{15}$ M. Martin, R. C. Martin, S. Allman, D. Brice, A. Wymore, and N. Andre, Spectrochim. Acta, Part B 114, 65 (2015).
${ }^{16}$ J. E. Barefield, E. J. Judge, K. R. Campbell, J. P. Colgan, D. P. Kilcrease, H. M. Johns, R. C. Wiens, R. E. McInroy, R. K. Martinez, and S. M. Clegg, Spectrochim. Acta, Part B 120, 1 (2016).

${ }^{17}$ M. Singh and A. Sarkar, Plasma Sci. Technol. 20, 125501 (2018).

${ }^{18}$ J. Blaise and L. J. Radziemski, J. Opt. Soc. Am. 66, 644 (1976).

${ }^{19}$ B. A. Palmer, R. A. Keller, and R. Engleman, Report No. LA 8251-MS, Los Alamos, New Mexico (1980).

${ }^{20}$ J. Blaise, J.-F. Wyart, J. Vergès, R. Engleman, B. A. Palmer, and L. J. Radziemski, J. Opt. Soc. Am. B 11, 1897 (1994).

${ }^{21}$ M. S. Finko and D. Curreli, Phys. Plasmas 25, 083112 (2018).

${ }^{22}$ P. J. Skrodzki, M. Burger, I. Jovanovic, M. C. Phillips, B. E. Brumfield, and S. S. Harilal, Opt. Lett. 43, 5118 (2018).

${ }^{23}$ R. McWhirter, in Plasma Diagnostic Technique, edited by R. H. Huddlestone and S. L. Leonard (Academic Press, New York, 1965).

${ }^{24}$ H. R. Griem, Spectral Line Broadening by Plasmas (Academic Press, New York, 1974).

${ }^{25}$ J. R. Wachter and D. A. Cremers, Appl. Spectrosc. 41, 1042 (1987).

${ }^{26}$ S. Harilal, P. Diwakar, N. LaHaye, and M. Phillips, Spectrochim. Acta, Part B 111, 1 (2015).

${ }^{27}$ P. Skrodzki, N. Shah, N. Taylor, K. Hartig, N. LaHaye, B. Brumfield, I. Jovanovic, M. Phillips, and S. Harilal, Spectrochim. Acta, Part B 125, 112 (2016).

${ }^{28}$ A. Sarkar, D. Alamelu, and S. K. Aggarwal, Appl. Opt. 47, G58 (2008).

${ }^{29} \mathrm{M}$. Burger and J. Hermann, Spectrochim. Acta, Part B122, 118 (2016).

${ }^{30}$ N. Konjević, A. Lesage, J. R. Führ, and W. L. Wiese, J. Phys. Chem. Ref. Data 19, 1307 (1990).

${ }^{31}$ N. Konjević, A. Lesage, J. R. Führ, and W. L. Wiese, J. Phys. Chem. Ref. Data 31, 819 (2002).

${ }^{32}$ A. Lesage, N. Astron. Rev. 52, 471 (2009).

${ }^{33}$ M. S. Finko, D. Curreli, D. G. Weisz, J. C. Crowhurst, T. P. Rose, B. Koroglu, H. B. Radousky, and M. R. Armstrong, J. Phys. D: Appl. Phys. 50, 485201 (2017).

${ }^{34}$ C. Corliss, J. Res. Natl. Bur. Stand. (U. S.) 80A, 429 (1976).

${ }^{35}$ S. S. Harilal, B. E. Brumfield, N. Glumac, and M. C. Phillips, Opt. Express 26, 20319 (2018).

${ }^{36}$ R. K. Singh and J. Narayan, Phys. Rev. B 41, 8843 (1990).

${ }^{37}$ T. Fujimoto, Plasma Spectroscopy, International Series of Monographs on Physics (Clarendon Press, 2004).

${ }^{38} \mathrm{R}$. G. Breene, The Shift and Shape of Spectral Lines (Pergamon Press, Oxford, 1961).

${ }^{39}$ M. A. Gigosos, J. Phys. D: Appl. Phys. 47, 343001 (2014).

${ }^{40}$ H. R. Griem, Plasma Spectroscopy (Academic Press, New York, 1964).

${ }^{41}$ J. Bernhardt, W. Liu, F. Théberge, H. Xu, J. Daigle, M. Châteauneuf, J. Dubois, and S. Chin, Opt. Commun. 281, 1268 (2008).

${ }^{42}$ J. T. Davies and J. M. Vaughan, Astrophys. J. 137, 1302 (1963).

${ }^{43}$ S. S. Harilal, J. Appl. Phys. 102, 123306 (2007).

${ }^{44}$ H. Bader, K. Rohr, and H. Weber, J. Phys. D: Appl. Phys. 13, L149 (1980).

${ }^{45}$ J. F. O'Hanlon, A User's Guide to Vacuum Technology, 3rd ed. (John Wiley \& Sons, Hoboken, New Jersey, 2003).

${ }^{46}$ G. Cristoforetti, A. D. Giacomo, M. Dell'Aglio, S. Legnaioli, E. Tognoni, V. Palleschi, and N. Omenetto, Spectrochim. Acta, Part B 65, 86 (2010).

${ }^{47}$ J. A. Aguilera, C. Aragón, and J. Bengoechea, Appl. Opt. 42, 5938 (2003).

${ }^{48}$ N. Konjević, Phys. Rep. 316, 339 (1999). 